Real Analysis Exchange

Vol. 23(2), 1997-1998, pp. 735-742

D. N. Sarkhel, Department of Mathematics, University of Kalyani, Kalyani 741235, West Bengal, India

\title{
ON THE APPROXIMATELY CONTINUOUS INTEGRALS OF BURKILL AND KUBOTA
}

\begin{abstract}
The exact relations between the approximately continuous Perron and Denjoy integrals of Burkill [1] and Kubota [5, 6] are re-established by rectifying the faulty proofs of Kubota, and the related questions of Gordon [3] are resolved completely.
\end{abstract}

In [2, p. 269] Gordon asked: Is there an approximately continuous integral that includes both the general Denjoy integral, $\mathcal{D}$-integral [10], and the approximately continuous Perron integral, AP-integral [1], of Burkill? This is a pertinent question since Tolstoff $[17$, p. 658] gave a function which is $\mathcal{D}$ integrable but not $A P$-integrable. But this question was resolved long ago by the author in the affirmative (see $[15$, p. 352], $[12,13]$ ), by introducing the $\left(T_{a} P\right)$ - and $\left(T_{a} D\right)$ - integrals where $T_{a}$ is the approximate limit process.

A still earlier solution is the approximately continuous Denjoy integral, $A D$-integral [5], and its equivalent the $A P^{*}$-integral [6], of Kubota. But in [3] Gordon asked the same question again, referring to a flaw in Kubota's proof [5, Theorem 2] that the $A D$-integral includes the $A P$-integral, and pointing out also certain flaws both in the indirect attempt of Lee [7] and in the direct attempt of Lin [8] to rectify Kubota's proof.

In this note we assume that the reader is familiar with the notions of $V B$, $A C, V B G$, Lusin's condition $(N)$, and approximate continuity and derivative [10]. Also, we refer the reader to $[15$, p. 337$]$ for the precise definitions of the following concepts: $A C$ above, $A C$ below, $A C G$ above, $A C G$ below, $A C G$, $(A C G)$ above, $(A C G)$ below, $(A C G),(V B G)$ and $(P A C)$. We mention that, a function $F$ is $A C G$ [resp. $V B G]$ on $[a, b]$ if $[a, b]$ is the union of a sequence of sets $\left\{E_{n}\right\}_{n=1}^{\infty}$ such that $F$ is $A C$ [resp. $\left.V B\right]$ on each $E_{n}$; if further each $E_{n}$ can be taken to be closed, then $F$ is said to be $(A C G)$ [resp. $(V B G)]$ on $[a, b]$. Note that $F$ is not required to be continuous on $[a, b]$. For $(P A C)$ we shall use the following equivalent definition:

Key Words: $A P$-integral, $A D$-integral, $A P^{*}$-integral, $(A C G)$ function

Mathematical Reviews subject classification: 26A39

Received by the editors April 4, 1997 
Definition (13, p. 296). A function $F:[a, b] \rightarrow \mathbb{R}$ is $(P A C)$ on $[a, b]$ if for every $\epsilon>0$ there exist an increasing sequence of sets $\left\{E_{n}\right\}$ with union $[a, b]$ and a sequence of positive numbers $\left\{r_{n}\right\}$, such that for every $n$ and every finite family $\left\{\left(a_{i}, b_{i}\right)\right\}$ of pairwise disjoint open intervals with endpoints in $E_{n}$ and with $\sum_{i}\left(b_{i}-a_{i}\right)<r_{n}$, we have $\sum_{i}\left|F\left(b_{i}\right)-F\left(a_{i}\right)\right|<\epsilon$.

Now, we find that Kubota used in fact the following type of fallacious arguments in three of his papers $[4,5,6]$ :

- If $\left\{M_{n}\right\}$ is a sequence of functions each of which is $(A C G)$ below on $[a, b]$, then there exists a sequence of closed sets $\left\{E_{k}\right\}$ with union $[a, b]$ such that each $M_{n}$ is $A C$ below on every $E_{k}$.

In the absence of other conditions, this is certainly not a valid argument (see [3, p. 837]). We will show, however, that Kubota's results [5, Theorem 2] and [6, Theorem 3.6] are correct in the context of these two papers. This resolves, in particular, the specific question 2 of Gordon [3, p. 838] in the affirmative. We make no attempt to rectify the proof of [4, Theorem 4.1], as it appears that Kubota abandoned [4] in favour of [5].

The related specific question 1 of Gordon [3, p. 838] is in essence the following: If a function $F$ satisfies the Lusin's condition $(N)$ and is approximately continuous and $V B G$ on $[a, b]$, then must $F$ be $(A C G)$ on $[a, b]$ ? The answer to this is an emphatic NO. Dwelling on this point, long ago the author constructed a function [15, Example 3.1, p. 342] which is approximately continuous and $(P A C)$, but not even $A C G$ below or $A C G$ above on $[a, b]$. It is to be noted that, by $[15$, Theorem 3.6], a function is $(P A C)$ on $[a, b]$ iff it satisfies the condition $(N)$ and is $(V B G)$ on $[a, b]$.

In this connection Gordon obtained a set of sufficient conditions [3, Theorem 4] for a function to be Baire* 1 on $[a, b]$. But this theorem is only a very special case of a more extensive result of the author [16, Theorem 2.1, p. 14]. It should be mentioned here that, Sargent [11, p. 117] calls a function $F$ continuous in the generalized sense, $(C G)$, on $[a, b]$ if $[a, b]$ is the union of a sequence of closed sets $\left\{E_{n}\right\}$ such that $F_{\mid E_{n}}$ is continuous for each $n$, and O'Malley [9] calls such a function Baire* 1.

As a solution to his opening question Gordon offered the $A K_{N}$-integral [3, p. 834], using the concept of $V B G_{N}$ functions. But there appears to be a serious oversight in his proof of the uniqueness of this integral, as it is not at all obvious that the difference of two $V B G_{N}$ functions always satisfies the Lusin's condition $(N)$. The difficulty lies in the use of the condition $V B G$ rather than $(V B G)$. But if the condition $V B G_{N}$ is replaced by $(V B G)_{N}$, then the resulting $A K_{N}$-integral reduces to the $\left(T_{a} P\right)$ and $\left(T_{a} D\right)$ - integrals $[12,13,15]$. 
We now prove the two results of Kubota. The derivative and the upper and lower derivatives, in the approximate sense, of a function $F$ will be denoted by $F_{a p}^{\prime}, \overline{A D F}$ and $\underline{A D F}$, respectively. The Lebesgue measure of a set $E$ will be denoted by $|E|$. We consider functions

$$
f:[a, b] \rightarrow[-\infty,+\infty] \text { and } M, m:[a, b] \rightarrow(-\infty,+\infty) .
$$

Burkill's $A P$-integral $[1, \S 3]$ can be defined as follows.

If $-\infty \neq \underline{A D} M(x) \geq f(x)$ for each $x$ in $[a, b], M(a)=0$, and $M$ is approximately continuous on $[a, b]$, then the function $M$ is called an AP-major function of $f$ on $[a, b]$.

If $\infty \neq \overline{A D} m(x) \leq f(x)$ for each $x$ in $[a, b], m(a)=0$, and $m$ is approximately continuous on $[a, b]$, then the function $m$ is called an AP-minor function of $f$ on $[a, b]$.

The function $f$ is said to be $A P$-integrable on $[a, b]$ if $f$ has both $A P$ major functions $M$ and $A P$-minor functions $m$ and $\inf \{M(b)\}=\sup \{m(b)\}$, and then this common finite value is defined to be the definite $A P$-integral of $f$ on $[a, b]$, denoted by $(A P) \int_{a}^{b} f$.

We remark that Burkill assumed $f$ to be measurable and finite almost everywhere. But these can be proved for $A P$-integrable $f$.

Kubota $[5, \S 3]$ defines the function $f$ to be $A D$-integrable on $[a, b]$ if there is a function $F$ which is approximately continuous and $(A C G)$ on $[a, b]$ and is such that $F_{a p}^{\prime}(x)=f(x)$ a.e. on $[a, b]$, and then $F(b)-F(a)$ is called the definite $A D$-integral of $f$ on $[a, b]$, denoted $(A D) \int_{a}^{b} f$.

Theorem 1. The AD-integral includes the AP-integral.

Proof. Let $f$ be $A P$-integrable on $[a, b]$. Then $[1, \S 4]$

$$
F(x)=(A P) \int_{a}^{x} f, \quad F(a)=0, a \leq x \leq b,
$$

is well defined, $F$ is approximately continuous on $[a, b]$, and $F_{a p}^{\prime}=f$ a.e. on $[a, b]$. So the proof will be complete once we can show that $F$ is $(A C G)$ on $[a, b]$. To this end, by $[15$, Theorem 3.5, p. 340] it is enough to show that $F$ is both $(P A C)$ and $(C G)$ on $[a, b]$.

To show that $F$ is $(P A C)$ on $[a, b]$ we use the method of proof of $[14$, Theorem 5.4, p. 39]. Given $\epsilon>0$, select an $A P$-major function $M$ and an $A P$-minor function $m$ of $f$ on $[a, b]$ such that

$$
H(b)<\epsilon \text { where } H=M-m .
$$


For each positive integer $n$ and each point $x$ in $[a, b]$, put

$$
A_{n}^{x}=\left\{y \in[a, b]: \frac{M(y)-M(x)}{y-x} \leq-n \text { or } \frac{m(y)-m(x)}{y-x} \geq n\right\} .
$$

Then let $E_{n}$ denote the set of points $x$ in $[a, b]$ such that

$$
\left|A_{n}^{x} \cap[u, v]\right|<\frac{1}{2}(v-u) \text { if } x \in[u, v] \text { and } v-u<\frac{1}{n} .
$$

Since $A_{n+1}^{x} \subseteq A_{n}^{x}, \underline{A D} M(x)>-\infty$ and $\overline{A D} m(x)<\infty$ for all $n, x$, clearly $\left\{E_{n}\right\}$ is an increasing sequence of sets with union $[a, b]$.

Now, if $u, v \in E_{n}$ and $0<v-u<1 / n$, then (1) implies that there are points $y \in(u, v) \backslash\left(A_{n}^{u} \cup A_{n}^{v}\right)$, and then we have

$$
\begin{aligned}
& M(y)-M(u)>-n(y-u), \quad m(y)-m(u)<n(y-u), \\
& M(v)-M(y)>-n(v-y), \quad m(v)-m(y)<n(v-y) .
\end{aligned}
$$

Since $M-F$ and $F-m$ are nondecreasing, we get

$$
\begin{gathered}
F(v)-F(u) \leq M(v)-M(u)=H(v)-H(u)+m(v)-m(u) \\
<H(v)-H(u)+n(v-u)
\end{gathered}
$$

and

$$
\begin{gathered}
F(u)-F(v) \leq m(u)-m(v)=H(v)-H(u)+M(u)-M(v) \\
<H(v)-H(u)+n(v-u) .
\end{gathered}
$$

Hence

$$
|F(v)-F(u)|<H(v)-H(u)+n(v-u) .
$$

Since $H$ is nondecreasing on $[a, b]$, it follows that for each $n$ and for every finite family of nonoverlapping intervals $\left\{\left[u_{i}, v_{i}\right]\right\}$ with endpoints in $E_{n}$ and with $\sum_{i}\left(v_{i}-u_{i}\right)<\epsilon / n$, we have

$$
\sum_{i}\left|F\left(v_{i}\right)-F\left(u_{i}\right)\right|<H(b)-H(a)+\epsilon=H(b)+\epsilon<2 \epsilon .
$$

Hence $F$ is $(P A C)$ on $[a, b]$.

Finally, since $M$ is approximately continuous and $\underline{A D} M>-\infty$ on $[a, b]$, as a special case of $[16$, Theorem 2.1, p. 14] $M$ is $(C G)$ on $[a, b]$. Also, $M-F$ is continuous on $[a, b]$ since it is nondecreasing and approximately continuous on $[a, b]$. Hence $F=M-(M-F)$ is $(C G)$ on $[a, b]$. This completes the proof. 
Remark. Since $F$ is $(A C G)$ on $[a, b]$, there is a sequence of closed sets $\left\{B_{n}\right\}$ with union $[a, b]$ such that $F$ is $A C$ on each $B_{n}$. Then for all $A P$-major functions $M$ and all $A P$-minor functions $m$ of $f$ on $[a, b]$, since $M-F$ and $F-m$ are nondecreasing on $[a, b]$, obviously each $M$ is $A C$ below and each $m$ is $A C$ above on every $B_{n}$. Thus the assertion of Kubota in his proof of [5, Theorem 2] is true, though not in his way.

Kubota's $A P^{*}$-integral $[6, \S 3]$ is defined as follows.

The function $M$ is called an $A P^{*}$-upper function of $f$ on $[a, b]$ if $M(a)=0$, $M$ is approximately continuous and $(A C G)$ below on $[a, b]$, and $M_{a p}^{\prime}(x) \geq f(x)$ a.e. on $[a, b]$.

The function $m$ is called an $A P^{*}$-lower function of $f$ on $[a, b]$ if $m(a)=0$, $m$ is approximately continuous and $(A C G)$ above on $[a, b]$, and $m_{a p}^{\prime}(x) \leq f(x)$ a.e. on $[a, b]$.

The function $f$ is said to be $A P^{*}$-integrable on $[a, b]$ if $f$ has both $A P^{*}$ upper functions $M$ and $A P^{*}$-lower functions $m$ on $[a, b]$ and $\inf \{M(b)\}=$ $\sup \{m(b)\}$, and then this common finite value is defined to be the definite $A P^{*}$-integral of $f$ on $[a, b]$, denoted $\left(A P^{*}\right) \int_{a}^{b} f$.

Theorem 2. The AD-integral is equivalent to the $A P^{*}$-integral.

Proof. This was proved by Kubota [6, Theorem 3.6]. But, as discussed above, there is a flaw in his proof that the $A D$-integral includes the $A P^{*}$ integral. So we will prove only this part.

Let $f$ be $A P^{*}$-integrable on $[a, b]$. Then $[6, \S 3]$

$$
F(x)=\left(A P^{*}\right) \int_{a}^{x} f, \quad F(a)=0, a \leq x \leq b,
$$

is well-defined, $F$ is approximately continuous on $[a, b]$, and $F_{a p}^{\prime}=f$ a.e. on $[a, b]$. So it remains only to show that $F$ is $(A C G)$ on $[a, b]$, that is that $F$ is both $(P A C)$ and $(C G)$ on $[a, b]$.

Given $\epsilon>0$, select an $A P^{*}$-upper function $M$ and an $A P^{*}$-lower function $m$ of $f$ on $[a, b]$ such that

$$
H(b)<\epsilon \text { where } H=M-m .
$$

Since $M$ is $(A C G)$ below and $m$ is $(A C G)$ above on $[a, b]$, we can find a sequence of closed sets $\left\{E_{n}\right\}$ with union $[a, b]$ such that, $M$ is $A C$ below and $m$ is $A C$ above on each $E_{n}$. Then for each $n$ there is a $\delta_{n}>0$ such that, for every finite family of nonoverlapping intervals $\left\{\left[a_{p}, b_{p}\right]\right\}$ with endpoints in $E_{n}$ and with $\sum_{p}\left(b_{p}-a_{p}\right)<\delta_{n}$, we have

$$
\sum_{p}\left(M\left(b_{p}\right)-M\left(a_{p}\right)\right)>-\frac{\epsilon}{2^{n}} \quad \text { and } \quad \sum_{p}\left(m\left(b_{p}\right)-m\left(a_{p}\right)\right)<\frac{\epsilon}{2^{n}} .
$$


Now, by [15, Lemma 2.1, p. 337], there is an increasing sequence of closed sets $\left\{F_{n}\right\}$ with union $[a, b]$ such that

$$
F_{n}=\cup_{k=1}^{n} F_{k n}, \quad F_{k n} \subseteq E_{k}, \quad \operatorname{dist}\left(F_{i n}, F_{j n}\right) \geq \frac{1}{n} \text { for } i \neq j .
$$

Consider any $n$ and any finite family of nonoverlapping intervals $\left\{\left[a_{p}, b_{p}\right]\right\}$ with endpoints in $F_{n}$ and with

$$
\sum_{p}\left(b_{p}-a_{p}\right)<\min \left\{\frac{1}{n}, \delta_{1}, \ldots, \delta_{n}\right\} .
$$

Since $\operatorname{dist}\left(F_{i n}, F_{j n}\right) \geq 1 / n$ for $i \neq j$, so for each $p$ both $a_{p}$ and $b_{p}$ must belong to precisely one of the sets $F_{k n}, k=1,2, \ldots, n$. Then, since $F_{k n} \subseteq E_{k}$, we clearly have

$$
\begin{gathered}
\sum\left(M\left(b_{p}\right)-M\left(a_{p}\right)\right)=\sum_{k=1}^{n} \sum_{a_{p} \in F_{k n}}\left(M\left(b_{p}\right)-M\left(a_{p}\right)\right)>\sum_{k=1}^{n} \frac{-\epsilon}{2^{k}}>-\epsilon, \\
\sum\left(m\left(b_{p}\right)-m\left(a_{p}\right)\right)=\sum_{k=1}^{n} \sum_{a_{p} \in F_{k n}}\left(m\left(b_{p}\right)-m\left(a_{p}\right)\right)<\sum_{k=1}^{n} \frac{\epsilon}{2^{k}}<\epsilon .
\end{gathered}
$$

Since $M-F, F-m$ and $H$ are nondecreasing, we get

$$
\begin{aligned}
\sum\left(F\left(b_{p}\right)-F\left(a_{p}\right)\right) & \leq \sum\left(M\left(b_{p}\right)-M\left(a_{p}\right)\right) \\
& =\sum\left(H\left(b_{p}\right)-H\left(a_{p}\right)\right)+\sum\left(m\left(b_{p}\right)-m\left(a_{p}\right)\right) \\
& <H(b)-H(a)+\epsilon=H(b)+\epsilon<2 \epsilon, \\
\sum\left(F\left(a_{p}\right)-F\left(b_{p}\right)\right) & \leq \sum\left(m\left(a_{p}\right)-m\left(b_{p}\right)\right) \\
& =\sum\left(H\left(b_{p}\right)-H\left(a_{p}\right)\right)+\sum\left(M\left(a_{p}\right)-M\left(b_{p}\right)\right) \\
& <H(b)-H(a)+\epsilon=H(b)+\epsilon<2 \epsilon .
\end{aligned}
$$

Thus $\left|\sum\left(F\left(b_{p}\right)-F\left(a_{p}\right)\right)\right|<2 \epsilon$. Hence, clearly, $\sum\left|F\left(b_{p}\right)-F\left(a_{p}\right)\right|<4 \epsilon$. Hence, as before, by definition $F$ is $(P A C)$ on $[a, b]$.

Finally, both $M-F$ and $F-m$ are continuous on $[a, b]$ since they are nondecreasing and approximately continuous on $[a, b]$. Since, further, $M$ is $A C$ below and $m$ is $A C$ above on each $E_{n}$, it follows readily from $F=M-$ $(M-F)=(F-m)+m$ that $F_{\mid E_{n}}$ is continuous for each $n$. Hence $F$ is $(C G)$ on $[a, b]$, which completes the proof. 


\section{References}

[1] J. C. Burkill, The approximately continuous Perron integral, Math. Zeit. 34 (1931), 270-278.

[2] R. A. Gordon, The integrals of Lebesgue, Denjoy, Perron and Henstock, Graduate Studies in Mathematics, AMS 4, Providence 1994.

[3] R. A. Gordon, Some comments on an approximately continuous Khintchine integral, Real Analysis Exchange 20 (1994-95), no. 2, 831-841.

[4] Y. Kubota, On the approximately continuous Denjoy integrals, Tôhoku Math. J. 15 (1963), 253-264.

[5] Y. Kubota, An integral of Denjoy type, Proc. Japan Acad. 40 (1964), $713-717$.

[6] Y. Kubota, An integral of Denjoy type. II, Proc. Japan Acad. 42 (1966), no. $7,737-742$.

[7] C. M. Lee, An analogue of the theorem of Hake-Alexandroff-Looman, Fund. Math. C (1978), 69-74.

[8] Y. Lin, A note on that Kubota's AD-integral is more general than Burkill's AP-integral, J. Mathematical Study 27 (1994), 116-120.

[9] R. J. O'Malley, Baire* 1, Darboux functions, Proc. Amer. Math. Soc. 60 (1976), 187-192.

[10] S. Saks, Theory of the integral, 2nd. rev. ed., vol. PWN, Monografie Matematyczne, Warsaw, 1937.

[11] W. L. C. Sargent, Some properties of $\mathcal{C}_{\lambda}$-continuous functions, J. London Math. Soc. 26 (1951), 116-121.

[12] D. N. Sarkhel, A wide Perron integral, Bull. Austral. Math. Soc. 34 (1986), 233-251.

[13] D. N. Sarkhel, A wide constructive integral, Math. Japonica 32 (1987), 295-309.

[14] D. N. Sarkhel and A. K. De, The proximally continuous integrals, J. Austral. Math. Soc. (Series A) 31 (1981), 26-45.

[15] D. N. Sarkhel and A. B. Kar, (PVB) functions and integration, J. Austral. Math. Soc. (Series A) 36 (1984), 335-353. 
[16] D. N. Sarkhel and P. K. Seth, On some generalized approximative relative derivatives, Rendi. Circ. Mat. Palermo (2) 35 (1986), 5-21.

[17] G. P. Tolstoff, Sur l'intégrale de Perron, Mat. Sb. 5 (1939), no. 47, 647660 .

The author wishes to thank the referee for the following additional references concerning the $A P^{*}$ - and $A D$-integrals.

[18] J. Ridder, Über approximativ stetige Denjoy-Integrale, Fund. Math. 21 (1933), 1-10.

[19] J. Ridder, Über die gegenseitigen Beziehungen vereschiedener approximativ stetigen Denjoy-Perron Integrale, Fund. Math. 22 (1934), 136-162.

The $A P^{*}$-integral was first introduced by Ridder in [18], (he calls it the $D_{4}$-integral in Definition 3, p. 5). It also appears in [19] (Definition 8, p. 149).

The $A D$-integral is in fact the $\beta$-Ridder integral introduced in [19] (Definition 7, p. 148). In fact in $[18$, p. 6$]$ Ridder asserts that the $A P^{*}$-integral is equivalent to the $A D$ - integral (with his notations of course) and makes the same faulty proof as Kubota.

The following related paper appeared after the acceptance of the present paper.

[20] C. M. Lee, Kubota's AD-integral is more general than Burkill's APintegral, Real Analysis Exchange 22 (1996-97), no. 1, 433-436. 